04.2

\title{
Первые наблюдения альфвеновских каскадов на токамаке Глобус-М2 и их применение для анализа минимума запаса устойчивости
}

\author{
(C) И.М. Балаченков ${ }^{1,2}$, Ю.В. Петров ${ }^{1}$, В.К. Гусев ${ }^{1}$, Н.Н. Бахарев ${ }^{1}$, В.В. Буланин ${ }^{1,2}$, В.И. Варфоломеев ${ }^{1}$, \\ Н.С. Жильцов ${ }^{1,2}$, Е.О. Киселёв ${ }^{1,2}$, Г.С. Курскиев ${ }^{1}$, В.Б. Минаев ${ }^{1}$, М.И. Патров ${ }^{1}$, А.В. Петров ${ }^{2}$, \\ А.М. Пономаренко ${ }^{2}$, Н.В. Сахаров ${ }^{1}$, А.Ю. Тельнова ${ }^{1}$, В.А. Токарев ${ }^{1}$, Н.А. Хромов ${ }^{1}$, П.Б. Щеголев ${ }^{1}$, \\ А.Ю. Яшин ${ }^{1,2}$ \\ ${ }^{1}$ Физико-технический институт им. А.Ф. Иофффе РАН, Санкт-Петербург, Россия \\ ${ }^{2}$ Санкт-Петербургский политехнический университет Петра Великого, Санкт-Петербург, Россия \\ E-mail: balachenkov@mail.ioffe.ru
}

Поступило в Редакцию 2 июля 2020 г.

В окончательной редакции 24 июля 2020 г.

Принято к публикации 6 августа 2020 г.

\begin{abstract}
На сферическом токамаке Глобус-М2 в режиме с магнитным полем 0.7 Т при помощи магнитных зондов, а также ряда других диагностик во время нейтральной инжекции на стадии роста тока зарегистрированы колебания магнитного поля в диапазоне $100-300 \mathrm{kHz}$ с нарастающей во времени частотой. Регистрировались моды с $n=1-3$ (тороидальное число) и $m=2-4$ (полоидальное число). Эти колебания идентифицированы как альфвеновские каскады. При помощи многоканального флуктуационного рефлектометра определена локализация обнаруженных мод вблизи нуля шира магнитного поля. Применение метода МГД-спектроскопии позволило определить изменение величины минимума коэффициента запаса устойчивости $\left(q_{\min }\right)$ во времени. Экспериментальные значения находятся в хорошем согласии с результатами расчета, полученными с помощью кода ASTRA.
\end{abstract}

Ключевые слова: токамак, магнитная диагностика, альфвеновские каскады, коэффициент запаса устойчивости, рефлектометр.

DOI: 10.21883/PJTF.2020.23.50338.18450

Глобус-М2 [1,2] - компактный сферический токамак с большим радиусом $R=0.36 \mathrm{~m}$ и малым радиусом $a=0.24 \mathrm{~m}$ (аспектное отношение $A=1.5$ ), который является модернизированной версией токамака Глобус-М [3]. Вследствие модернизации в токамаке могут быть достигнуты тороидальное магнитное поле до $1 \mathrm{~T}$ и ток по плазме до $500 \mathrm{kA}$. Недавно [4] на токамаке Глобус-М2 была проведена серия экспериментов с тороидальным магнитным полем $0.7 \mathrm{~T}$, при этом во время импульса нейтральной инжекции $(28 \mathrm{keV}$, $0.85 \mathrm{MW})$ на стадии подъема тока при помощи массива магнитных зондов были зарегистрированы периодически возникающие продолжительные возмущения магнитного поля с нарастающей во времени частотой в диапазоне $100-300 \mathrm{kHz}$ (рис. 1,a). Аналогичные сигналы также удалось зарегистрировать при помощи других диагностик: подвижного ленгмюровского зонда, диагностики допплеровского обратного рассеяния и флуктуационного рефлектометра, которые были установлены в экваториальной плоскости со стороны слабого магнитного поля. На рис. 1, a показана спектрограмма колебаний магнитного потока, наведенного на зонд Мирнова, на рис. $1, b-$ спектрограмма колебаний потенциала ленгмюровского зонда [5], на рис. 1, $c$ - спектрограмма колебаний скорости вращения, полученная методом допплеровского обратного рассеяния [6]. Описанное поведение характерно для так называемых мод на обращенном шире (reversed shear Alfven eigenmode, RSAE) или альф- веновских каскадов (Alfven cascade, AC) [7,8]. Несмотря на то что каскады многократно регистрировались как на классических токамаках с большим аспектным отношением, таких как JET [7], так и на сферических, например NSTX $[9,10]$, на токамаке Глобус-М они ранее не наблюдались. Одна из возможных причин состоит в том, что необходимым условием возникновения альфвеновских каскадов является низкое значение $\beta_{e}=8 \pi p_{e} / B_{0}^{2}\left(p_{e}-\right.$ газокинетическое давление электронов, $B_{0}$ - поле на магнитной оси) [9], чего не удавалось достичь в разрядах с нейтральной инжекцией при полях $0.3-0.5$ T. При увеличении тороидального магнитного поля до 0.6-0.7 Т в токамаке Глобус-М2 стало возможным получить разряды с низкими значениями $\beta_{e}$, в которых и обнаружились альфвеновские каскады. Другая возможная причина состоит в улучшении удержания быстрых частиц, „раскачивающих“ неустойчивость, которое связано с увеличением тока по плазме.

Альфвеновский каскад - это собственная альфвеновская мода, которая при низких значениях $\beta_{e}$ подчиняется дисперсионному соотношению для сдвиговой альфвеновской волны (shear Alfven wave) $[8,9]: \omega^{2}=k_{\|}^{2} V_{A}^{2}$, где $k_{\|}=(m-n q) / q R$ - компонента волнового вектора в направлении магнитного поля, $V_{A}=B / \sqrt{4 \pi \rho_{i}}-$ альфвеновская скорость, $q$ - коэффициент запаса устойчивости, $R$ - большой радиус, $m$ и $n-$ соответственно полоидальный и тороидальный номера мод, $B$ локальная величина магнитного поля, $\rho_{i}-$ массовая 
плотность плазмы. Как правило, альфвеновские колебания возбуждаются за счет резонанса с быстрыми (надтепловыми) частицами, в роли которых на токамаке Глобус-М2 выступают быстрые ионы, образовавшиеся в результате ионизации атомов нейтрального пучка [11]. При быстром нарастании тока из-за скинового
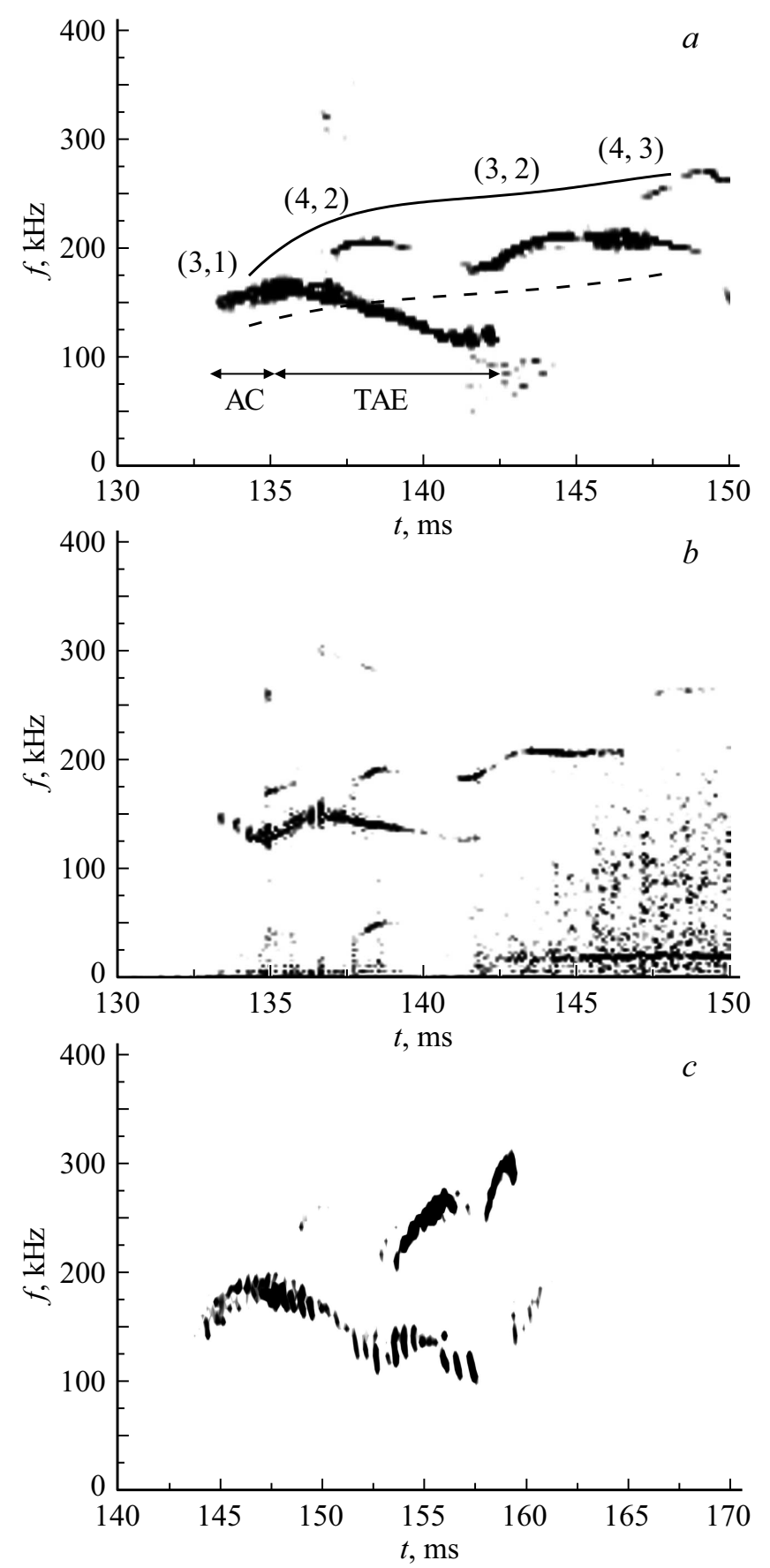

Рис. 1. Наблюдение альфвеновских каскадов на токамаке Глобус-М2. $a-$ спектрограмма сигнала магнитного зонда, разряд № $38035,130-165 \mathrm{~ms} ; b-$ спектрограмма колебаний потенциала подвижного ленгмюровского зонда, разряд № 38782, 130-150 ms; c - спектрограмма колебаний допплеровского частотного сдвига излучения на частоте $48 \mathrm{GHz}$, разряд № 38390, 140-170 ms.
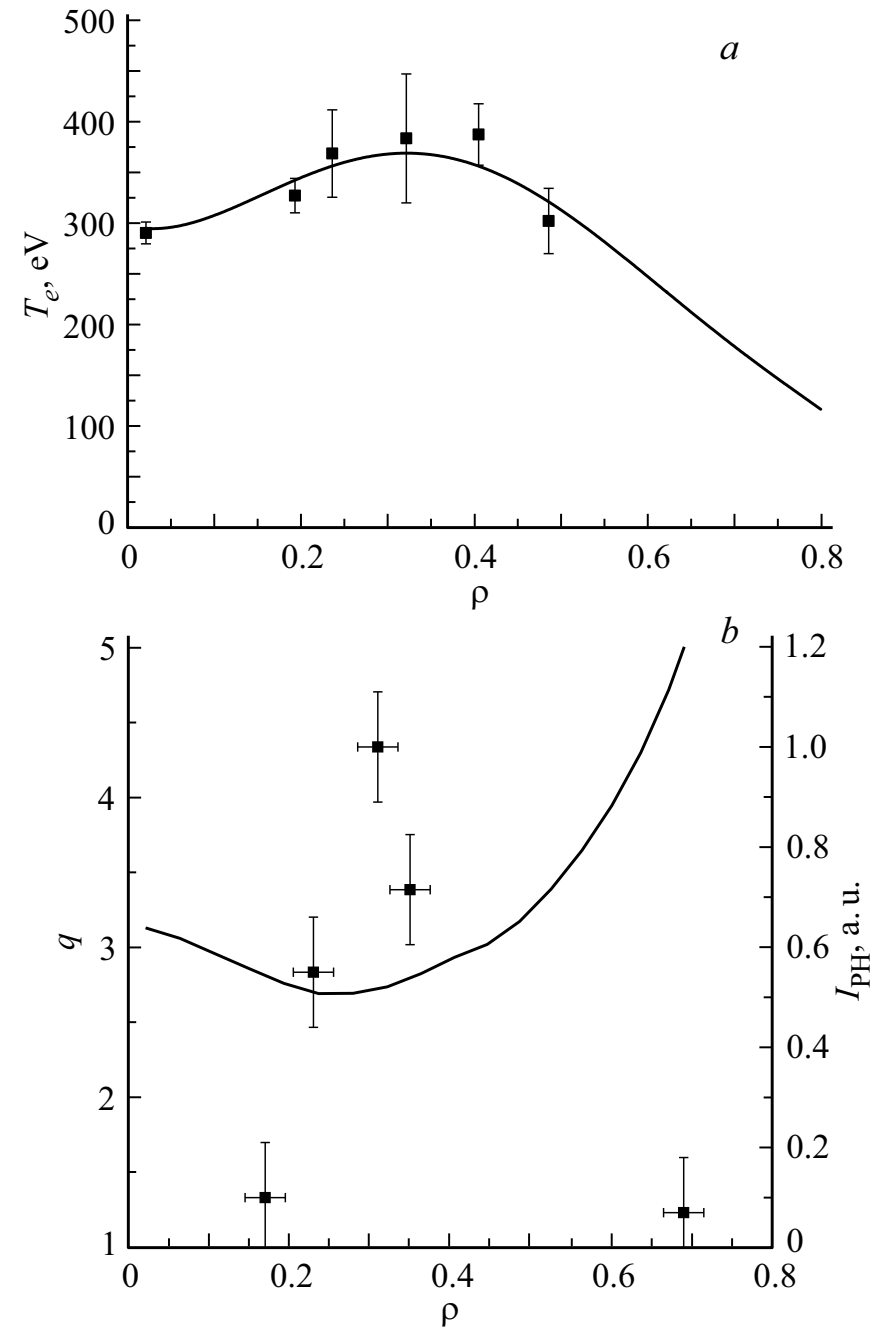

Рис. 2. Определение пространственной локализации альфвеновских каскадов. $a-$ профиль электронной температуры, разряд № 38035, $134 \mathrm{~ms}$. Квадраты соответствуют экспериментальным точкам по данным диагностики ТР. Сплошная линия - профиль, восстановленный при помощи кода ASTRA. $b$ - пространственная локализация альфвеновских каскадов. Квадраты - амплитуда колебаний фазы отраженного сигнала в относительных единицах, разряд № 39060, $140 \mathrm{~ms}$. Сплошная линия - расчетный профиль $q$, полученный при помощи кода ASTRA разряд № 38035, 134 ms.

эффекта в разряде может формироваться обращенный профиль $q$ в центральной области плазменного шнура с минимальной величиной $q_{\min }$ не на оси шнура, о чем можно судить исходя из формы профиля электронной температуры (рис. 2,a). При инжекции нейтрального пучка на стадии роста тока такой профиль может „замораживаться“ на некоторое время благодаря нецентральному вкладу мощности нагрева. Это в свою очередь создает благоприятные условия для развития каскада, поскольку он может существовать сколь-либо продолжительное время, не подвергаясь затуханию в альфвеновском континууме, только вблизи некоторой области, внутри которой происходит обращение в нуль 
магнитного шира, $(r / q)(d q / d r)[12,13]$. По мере роста тока происходит уменьшение величины $q_{\min }$, из-за чего происходит линейный рост частоты каскада. В связи с этим обнаружение альфвеновских каскадов открывает новые возможности для диагностики минимума коэффициента запаса устойчивости $q_{\min }$ путем анализа спектров сигналов магнитных измерений (так называемая МГД-спектроскопия $[9,10])$. Более высоким значениям $\beta_{e}$ соответствует более сложное дисперсионное соотношение, включающее „зацепление“ альфвеновской и геодезической акустической моды (geodesic-acoustic mode, GAM) [8,9]:

$$
\omega_{\mathrm{AC}}^{2}=k_{\|}^{2} V_{A}^{2}+\frac{2 c_{s}^{2}}{R^{2}} .
$$

В соотношении (1) $c_{s}^{2}=T_{e}\left(1+7 T_{i} / 4 T_{e}\right) / m_{i}-$ ионно-звуковая скорость, $T_{i}$ и $T_{e}-$ температура ионов и электронов соответственно. Первое слагаемое в выражении (1), как и раньше, определяется дисперсионным соотношением для сдвиговой альфвеновской волны [12], второе - частотой GAM [14]. Необходимо отметить, что частота GAM в дисперсионном соотношении является лишь нижним теоретическим пределом частоты каскада, который отражает взаимодействие альфвеновской моды с акустическими колебаниями. Геодезическая акустическая мода сама по себе в условиях эксперимента по наблюдению каскадов не регистрировалась. При появлении резонансной магнитной поверхности с $q=m / n$ первый член в этом выражении обращается в нуль, это следует из периодических граничных условий, накладываемых на продольное волновое число в тороидальной геометрии токамака. Таким образом, минимальная частота альфвеновского каскада определяется вторым членом дисперсионного соотношения, т.е. частотой GAM. Далее уменьшение коэффициента запаса устойчивости $q$ приводит к линейному росту частоты каскада, который происходит до того момента, пока частота каскада не достигает частоты тороидальной альфвеновской моды (toroidicity-induced Alfven eigenmode, TAE), которая определяется выражением $[9,12]$ :

$$
\omega_{\mathrm{TAE}}=\frac{V_{A}}{2 q R},
$$

и не происходит трансформации моды.

Пока $q_{\min }$ продолжает уменьшаться существует возможность для развития мод с другими номерами $m$ и $n$ на большей частоте. За спадающие частотные ветви на рис. 1 (например, для моды $n=1$ на рис. $1, a$ co 135 по $143 \mathrm{~ms}$ ) отвечает снижение альфвеновской скорости $V_{A}$ на фоне роста концентрации для тороидальной альфвеновской моды, в которую трансформируются моды каскада. Частота каскада оказывается ограниченной двумя значениями [10]: минимальной частотой $f_{\min }=f_{\mathrm{GAM}}=c_{s} / \sqrt{2} \pi R$ (штриховая кривая на рис. $\left.1, a\right)$ и максимальной частотой $f_{\max }^{2}=f_{\min }^{2}+f_{\mathrm{TAE}}^{2}$ (сплошная кривая на рис. $1, a)$.
Для нахождения $q_{\min }$ требуется определить пространственную структуру возмущения. Если тороидальное волновое число $n$ довольно легко определяется по сдвигу фаз сигналов четырех магнитных зондов, расположенных на равных расстояниях вдоль тороидального обхода токамака, то установить полоидальное число $m$ по сдвигу фаз или корреляционными методами $[15,16]$ оказывается затруднительно ввиду того, что в пределах каскада частота сигнала меняется достаточно быстро. Определить волновые числа $m$ для каждой из мод каскада можно, применяя подход, изложенный в работах $[9,10]$, основанный на том, что каждый следующий каскад появляется при прохождении $q_{\min }$ целочисленного значения и величина $q_{\min }$ монотонно уменьшается при увеличении тока в разряде. Например, для разряда № 38035 (рис. $1, a$ ) известно, что для первой моды $n=1$, для следующих двух $n=2$ и для последней $n=3$. Кроме того, на $137 \mathrm{~ms}$ разряда одновременно регистрируются моды с $n=1$ и 2. Единственным набором $(m, n)$, отвечающим всем перечисленным требованиям, является последовательность $(3,1),(4,2),(3,2),(4,3)$.

Установив волновые числа каскадов и имея данные о профилях концентрации и температуры, измеренные при помощи диагностики томсоновского рассеяния (ТР) [17], можно вычислить значения $q_{\min }$, воспользовавшись выражением, полученным из формулы (1):

$$
q_{\min }=\frac{m V_{A}}{n V_{A}+R \sqrt{\omega^{2}-\omega_{\min }^{2}}} .
$$

Для вычисления $q_{\min }$ использовалось предположение о центральной локализации альфвеновской неустойчивости, поскольку обращение профиля $q$, как правило, происходит вблизи центра плазменного шнура, а именно там, где достигается максимум температуры.

Позднее локализация альфвеновских каскадов была установлена экспериментально с помощью многочастотного рефлектометра. Зондирование излучением $O$-моды осуществлялось на частотах $39,50,55,60$ и $65 \mathrm{GHz}$. Использовалось квадратурное детектирование, что позволяло определять фазу отраженного сигнала. Регистрировались колебания фазы на частоте альфвеновских колебаний, которые определялись деформациями профиля плотности плазмы под действием альфвеновских колебаний магнитного поля. В результате была получена зависимость амплитуды колебаний фазы от малого радиуса в разряде № 39060, которая представлена на рис. $2, b$. Максимум амплитуды колебаний фазы отраженного сигнала при этом находится вблизи $\rho=0.3$.

Результаты, полученные методом МГД-спектроскопии, сравнивались с расчетом, проведенным с помощью кода ASTRA [18]. Для определения $q_{\min }$ был проведен нестационарный расчет. В качестве входных данных использовались профили электронной температуры и концентрации, полученные экспериментально при помощи диагностики ТР. Ионная температура рассчитывалась 


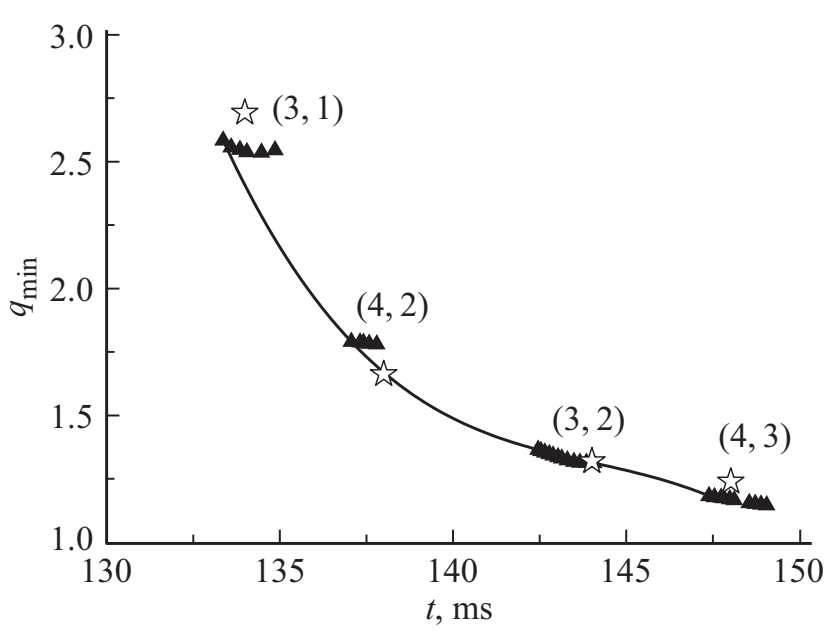

Рис. 3. Эволюция $q_{\min }$ в разряде № 38035 (треугольники данные МГД-спектроскопии, звездочки - результат расчета с помощью кода ASTRA).

$\mathrm{V}$

на основании неоклассических коэффициентов температуропроводности [19] и сравнивалась с измерениями анализатора атомов перезарядки. Для установления эффективного заряда использовалась специальная диагностика, определяющая $Z_{e f f}$ по интенсивности тормозного излучения [20]. В каждой точке измерений диагностики ТР проводился расчет профиля $q$ и магнитного шира. Значения $q$ вблизи нуля магнитного шира (звездочки на рис. 3) сравнивались со значениями $q_{\min }$, полученными из анализа частоты АС (треугольники на рис. 3), и продемонстрировали хорошее соответствие. Радиус обращения $q$, полученный в результате моделирования (разряд № 38035), оказался близок к радиусу, при котором с помощью рефлектометра была определена пространственная локализация альфвеновского каскада в разряде № 39060 (рис. 2,b). Из-за отсутствия всей полноты данных диагностик для каждого из разрядов сравнение проведено для различных, хотя и близких по параметрам разрядов. В обоих разрядах установлен режим, в котором возникают альфвеновские каскады, однако фаза подъема тока в разряде № 39060 сдвинута по времени, поэтому профиль $q$ для разряда № 38035 и профиль величины фазового сдвига сигнала рефлектометра приведены для разных моментов времени, соответствующих появлению моды $n=1$. Таким образом, совокупность экспериментальных и расчетных данных указывает на адекватность примененной нами модели развития АС в токамаке Глобус-М2 и открывает возможность использования МГД-спектроскопии для определения $q_{\min }$ в разрядах с инжекцией нейтрального пучка на стадии роста тока.

\section{Финансирование работы}

Эксперименты проводились на уникальной научной установке „Сферический токамак Глобус-М“, входящей в состав Центра коллективного пользования „Материаловедение и диагностика в передовых технологиях“ (идентификатор проекта RFMEFI62119X0021). Измерения основных параметров плазмы выполнены в рамках госзадания Министерства образования и науки РФ. Рефлектометрические измерения для определения пространственной локализации альфвеновских каскадов, магнитные измерения и моделирование коэффициента запаса устойчивости кодом ASTRA проведены при финансовой поддержке Российского научного фонда (проект № 1712-01177-П).

\section{Конфликт интересов}

Авторы заявляют, что у них нет конфликта интересов.

\section{Список литературы}

[1] Minaev V.B., Gusev V.K., Sakharov N.V., Varfolomeev V.I., Bakharev N.N., Belyakov V.A., Bondarchuk E.N., Brunkov P.N., Chernyshev F.V., Davydenko V.I., Dyachenko V.V., Kavin A.A., Khitrov S.A., Khromov N.A., Kiselev E.O., Konovalov A.N., Kornev V.A., Kurskiev G.S., Labusov A.N., Melnik A.D., Mineev A.B., Mironov M.I., Miroshnikov I.V., Patrov M.I., Petrov Yu.V., Rozhansky V.A., Saveliev A.N., Senichenkov I.Yu., Shchegolev P.B., Shcherbinin O.N., Shikhovtsev I.V., Sladkomedova A.D., Solokha V.V., Tanchuk V.N., Telnova A.Yu., Tokarev V.A., Tolstyakov S.Yu., Zhilin E.G. // Nucl. Fusion. 2017. V. 57. P. 066047. https://doi.org/10.1088/1741-4326/aa69e0

[2] Gusev V.K., Bakharev N.N., Belyakov V.A., Ber B.Ya., Bondarchuk E.N., Bulanin V.V., Bykov A.S., Chernyshev F.V., Demina E.V., Dyachenko V.V., Goncharov P.R., Gorodetsky A.E., Gusakov E.Z., Iblyaminova A.D., Ivanov A.A., Irzak M.A., Kaveeva E.G., Khitrov S.A., Khokhlov M.V., Khromov N.A., Kolmogorov V.V., Kornev V.A., Krasnov S.V., Kurskiev G.S., Labusov A.N., Lepikhov S.A., Litunovsky N.V., Mazul I.V., Melnik A.D., Mikov V.V., Minaev V.B., Mineev A.B., Mironov M.I., Miroshnikov I.V., Mukhin E.E., Novokhatsky A.N., Ovsyannikov A.D., Patrov M.I., Petrov A.V., Petrov Yu.V., Rozhansky V.A., Sakharov N.V., Saveliev A.N., Senichenkov I.Yu., Sergeev V.Yu., Shchegolev P.B., Shcherbinin O.N., Shikhovtsev I.V., Tanaev V.S., Tanchuk V.N., Tolstyakov S.Yu., Varfolomeev V.I., Vekshina E.O., Voronin A.V., Voskoboinikov S.P., Wagner F., Yashin A.Yu., Zadvitskiy G.V., Zakharov A.P., Zalavutdinov R.Kh., Zhilin E.G. // Nucl. Fusion. 2015. V. 55. P. 104016. https://doi.org/10.1088/0029-5515/55/10/104016

[3] Гусев В.К., Голант В.Е., Гусаков Е.З., Дьяченко В.В., Ирзак М.А., Минаев В.Б., Мухин Е.Е., Новохацкий А.Н., Подушникова К.А., Раздобарин Г.Т., Сахаров Н.В., Трегубова Е.Н., Узлов В.С., Щербинин О.Н., Беляков В.А., Кавин А.А., Косиов Ю.А., Кузьмин Е.Г., Сойкин В.Ф., Кузнецов Е.А., Ягнов В.А. // ЖТФ. 1999. Т. 69. В. 9. С. 58 62. https://journals.ioffe.ru/articles/36172 
[4] Бахарев Н.Н., Балаченков И.М., Варфоломеев В.И., Воронин А.В., Гусев В.К., Дьяченко В.В., Ильясова М.В., Киселев Е.О., Коновалов А.Н., Курскиев Г.С., Мельник А.Д., Минаев В.Б., Мирошников И.В., Новохачкий А.Н., Патров М.И., Петров Ю.В., Сахаров Н.В., Скрекель О.М., Тельнова А.Ю., Токарев В.А., Толстяков С.Ю., Тюхменева Е.А., Хилькевич Е.М., Хромов Н.А., Чернышев Ф.В., Чугунов И.Н., Шевелев А.Е., Щеголев П.Б. // Физика плазмы. 2020. Т. 46. В. 7. С. 579-587. https://doi.org/10.31857/S036729212007001X

[5] Tokarev V.A., Gusev V.K., Khromov N.A., Patrov M.I., Petrov Yu.V., Varfalomeev V.I. // J. Phys.: Conf. Ser. 2018. V. 1094 P. 012003. https://doi.org/10.1088/1742-6596/1094/1/012003

[6] Буланин В.В., Гусев В.К., Курскиев Г.С., Минаев В.Б., Патров М.И., Петров А.В., Петров Ю.В., Яшин А.Ю. // Письма в ЖТФ. 2019. Т. 45 В. 21. С. 44-47. DOI: 10.21883/PJTF.2019.21.48474.17982

[7] Sharapov S.E., Alper B., Berk H.L., Borba D.N., Breizman B.N., Challis C.D., Fasoli A., Hawkes N.C., Hender T.C., Mailloux J., Pinches S.D., Testa D., and EFDAJET work programme // Phys. Plasmas. 2002. V. 9. P. 20272036. https://doi.org/10.1063/1.1448346

[8] Breizman B.N., Pekker M.S., Sharapov S.E., and JET EFDA contributors // Phys. Plasmas. 2005. V. 12. P. 112506. https://doi.org/10.1063/1.2130692

[9] Fredrickson E.D., Crocker N.A., Gorelenkov N.N., Heidbrink W.W., Kubota S., Levinton F.M., Yuh H., Menard J.E., Bell R.E. // Phys. Plasmas. 2008. V. 14. P. 102510. https://doi.org/10.1063/1.2768038

[10] Crocker N.A., Fredrickson E.D., Gorelenkov N.N., Kramer G.J., Darrow D.S., Heidbrink W.W., Kubota S., Levinton F.M., Yuh H., Menard J.E., LeBlanc B.P., Bell R.E. // Phys. Plasmas. 2008. V. 15. P. 102502. https://doi.org/10.1063/1.2993182

[11] Bakharev N.N., Chernyshev F.V., Goncharov P.R., Gusev V.K., Iblyaminova A.D., Kornev V.A., Kurskiev G.S., Melnik A.D., Minaev V.B., Mironov M.I., Patrov M.I., Petrov Yu.V., Sakharov N.V., Shchegolev P.B., Tolstyakov S.Yu., Zadvitskiy G.V. // Nucl. Fusion. 2015. V. 55. P. 043023.

[12] Heidbrink W.W. // Phys. Plasmas. 2008. V. 15. P. 055501. https://doi.org/10.1063/1.2838239

[13] Курскиев Г.С., Гусев В.К., Толстяков С.Ю., Березуцкий А.А., Буланин В.В., Варфоломеев В.И., Кочергин М.М., Минаев В.Б., Мухин Е.Е., Патров М.И., Петров А.В., Петров Ю.В., Сахаров Н.В., Семёнов В.В., Яшин А.Ю., Хромов Н.А. // Письма в ЖТФ. 2011. Т. 37. B. 23. C. 82-90. https://journals.ioffe.ru/articles/14381

[14] Itoh K., Hallatschek K., Itoh S.-I. // Plasma Phys. Control. Fusion. 2005. V. 47. P. 451-458. https://doi.org/10.1088/0741-3335/47/3/004

[15] Melnikov A.V., Markovic T., Eliseev L.G., Adámek J., Aftanas M., Bilkova P., Boehm P., Gryaznevich M., Imrisek M., Lysenko S.E., Medvedev S.Yu., Panek R., Peterka M., Seidl J., Stefanikova E., Stockel J., Weinzettl V., and the COMPASS team // Plasma Phys. Control. Fusion. 2015. V. 57. P. 065006. https://doi.org/10.1088/0741-3335/57/6/065006

[16] Balachenkov I.M., Patrov M.I., Petrov Yu.V., Tukachinsky A.S. // J. Phys.: Conf. Ser. 2019. V. 1400. P. 077016. https://doi.org/10.1088/1742-6596/1400/7/077016
[17] Курскиев Г.С., Толстяков С.Ю., Березуиякий А.А., Гусев В.К., Кочергин М.М., Минаев В.Б., Мухин Е.E., Патров М.И., Петров Ю.В., Сахаров Н.В., Семёнов В.В., Чернаков П.В. // ВАНТ. Сер. Термоядерный синтез. 2012. B. 2 C. $81-88$. https://www.elibrary.ru/item.asp?kid=17918179

[18] Pereverzev G.V., Yushmanov P.N. ASTRA (Automated System for TRansport Analysis). Max-Plank report. 2002. IPP 5/98. https://www.osti.gov/etdeweb/biblio/20245330

[19] Telnova A.Yu., Kurskiev G.S., Miroshnikov I.V., Sakharov N.V., Kiselev E.O., Larionova M.M., Bakharev N.N., Larionova D.M., Gusev V.K., Khromov N.A., Minaev V.B., Patrov M.I., Petrov Yu.V., Sladkomedova A.D., Shchegolev P.B., Tokarev V.A., Tolstyakov S.Yu., Tukhmeneva E.A. // Plasma Phys. Control. Fusion. 2020. V. 62 P. 045011. https://doi.org/10.1088/1361-6587/ab6da5

[20] Tukhmeneva E.A., Tolstyakov S.Yu., Kurskiev G.S., Gusev V.K., Minaev V.B., Petrov Yu.V., Sakharov N.V., Telnova A.Yu., Bakharev N.N., Shchegolev P.B., Kiselev E.O. // Plasma Sci. Technol. 2019. V. 21. P. 105104. https://doi.org/10.1088/2058-6272/ab305f 